Musées, Patrimoine et Culture scientifiques et techniques

$151 \mid 2014$

janvier-février 2014

\title{
La conservation préventive, une perpétuelle actualité
}

\section{Juliette Barbarin}

\section{(Q) OpenEdition \\ Journals}

Édition électronique

URL : http://journals.openedition.org/ocim/1306

DOI : $10.4000 /$ ocim. 1306

ISSN : 2108-646X

Éditeur

OCIM

Édition imprimée

Date de publication : 1 février 2014

ISSN : 0994-1908

Référence électronique

Juliette Barbarin, «La conservation préventive, une perpétuelle actualité », La Lettre de l'OCIM [En

ligne], 151 | 2014, mis en ligne le 31 janvier 2015, consulté le 30 avril 2019. URL : http://

journals.openedition.org/ocim/1306 ; DOI : 10.4000/ocim.1306

Ce document a été généré automatiquement le 30 avril 2019

Tous droits réservés 


\title{
La conservation préventive, une perpétuelle actualité
}

\author{
Juliette Barbarin
}

1 Le musée de la ville de Chalon-sur-Saône, installé dans les locaux rénovés d'une école de dessin, est inauguré en 1866 sous l'impulsion de sociétés savantes. Il recèle alors des collections d'archéologie, de beaux-arts et de sciences naturelles. Son directeur et fondateur collecte également les héliographies et le premier appareil photographique de Niépce ainsi qu'une abondante documentation. Résolument encyclopédique, le musée prend le nom de Vivant Denon en 1895, en hommage au premier directeur du musée du Louvre. Sa dernière rénovation remonte à 1958, lorsque le conservateur de l'époque profite d'une dernière extension pour redéployer les collections de la Préhistoire au XX siècle.

2 Dès le début des années 1970, la ville de Chalon-sur-Saône décide de se doter d'un musée d'envergure consacré à la photographie en hommage à Nicéphore Niépce. 


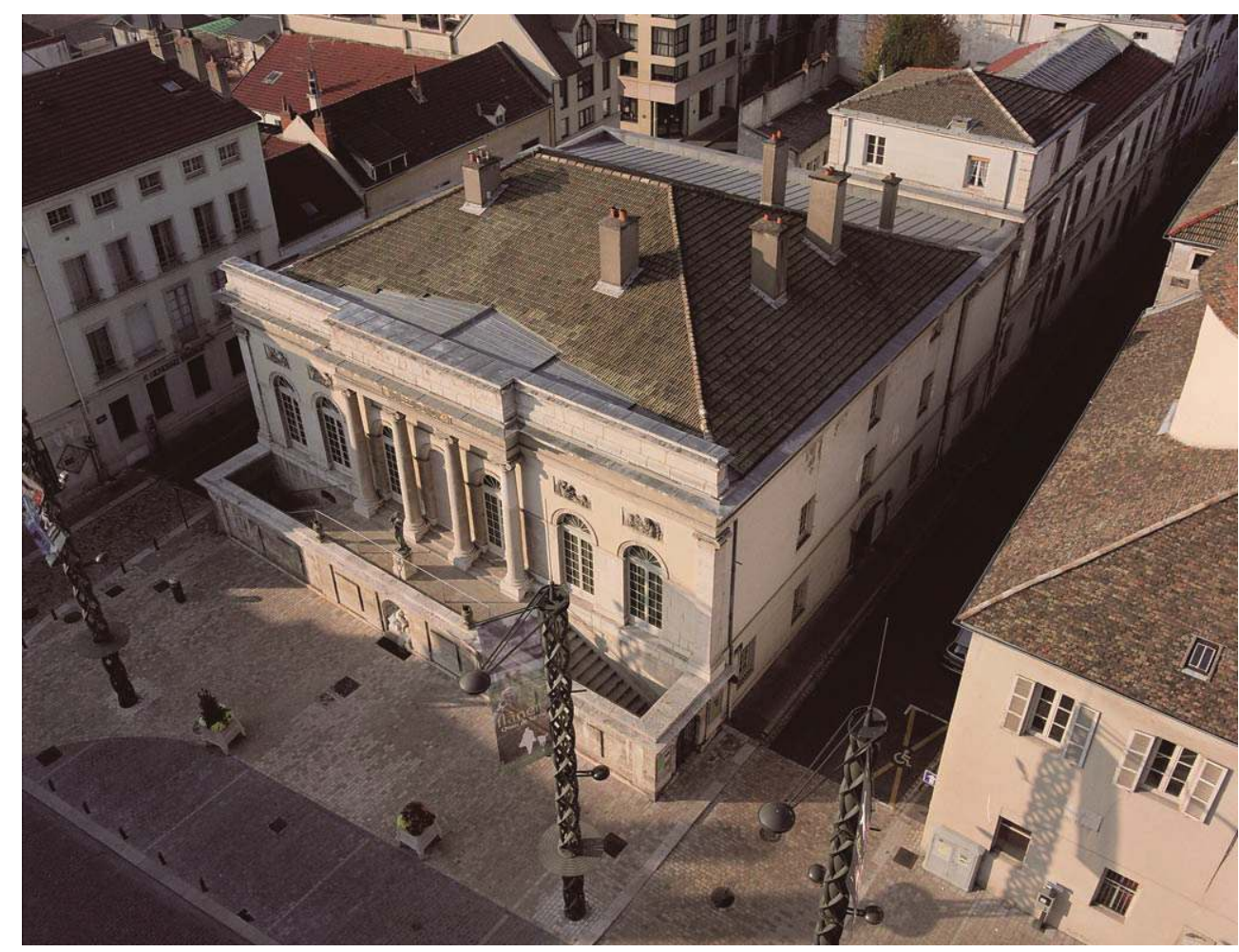

Le musée Vivant Denon, 2004

(c) Ville de Chalon-sur-Saône

En 2000, la création d'une direction unique des musées chalonnais permet de réfléchir à une réorganisation progressive du musée Denon, en accord avec le projet scientifique et culturel du musée Niépce.

Le diagnostic posé alors sur le musée Denon faisait le constat d'une accumulation d'œuvres dans un bâtiment peu entretenu. Avant d'analyser le potentiel des collections, il est apparu indispensable de concevoir un plan de conservation préventive pour les rendre accessibles et professionnaliser une équipe autour de la matérialité des collections 2. Il a donc été fait appel au département Conservation préventive du Centre de Recherche et de Restauration des Musées de France (C2RMF) pour un diagnostic précis et l'établissement d'un cahier des charges. L'étude a mis en évidence trois priorités dont la plus urgente consistait à repenser la localisation des collections et aménager des espaces de stockage. Les agents du musée ont donc été formés et accompagnés par une équipe de restaurateurs spécialisés pendant dix ans pour traiter 10000 objets de la section BeauxArts et commencer la sauvegarde des collections archéologiques.

Une première pierre pour un long chemin : un état des lieux et une étude en conservation préventive

Il est toujours difficile de savoir comment un projet, a fortiori pluriannuel, va évoluer et même aboutir, au gré des événements, des possibilités financières, des acteurs et des décideurs... Mais il était certain en 2001 que celui qui allait se mettre en place au musée Denon de Chalon-sur-Saône démarrait sur de bonnes bases, ce qui assurait la première garantie de sa réussite possible.

La situation n'était pourtant pas facile avec de nombreux facteurs en jeu : musée hérité d'une gestion des années 1970-1980 avec une distinction nette entre les 
collections de beaux-arts et celles d'archéologie pourtant présentes dans un même bâtiment, une attention à la conservation et au rangement des collections telle qu'on pouvait la concevoir à l'époque (réserves dispersées entre différents espaces non adaptés, conditionnement...) et un état sanitaire des bâtiments relativement préoccupant.

Alors qu'on aurait pu être tenté de résoudre au plus pressé ou au plus visible, Juliette Barbarin avec l'appui de François Cheval (conservateur du musée Nicéphore Niépce) a pris la décision de la réflexion, de prendre du temps et d'aborder le dossier Denon dans sa globalité et non, malgré certains facteurs locaux, d'apporter des réponses partielles en attendant certaines décisions.

Le choix fut simple et clair : offrir prioritairement de bonnes conditions de conservation aux collections et privilégier toute action le permettant, dans le cadre d'un plan de conservation préventive.

C'est dans ce contexte que fut demandée une première mission au C2RMF, département de Conservation préventive (9 février 2001). Elle attira l'attention sur un état satisfaisant des collections mais une inadaptation des espaces et des équipements et surtout un mauvais état sanitaire du bâtiment. La préconisation fut de faire réaliser une étude en conservation préventive afin d'identifier plus précisément certains états matériels des collections et de proposer des interventions, de définir un plan de travail hiérarchisé et pour lequel le personnel du musée pourrait être associé après avoir été formé. La mission soulignait aussi le besoin d'une implication forte des services techniques de la ville afin d'étudier et de résoudre des désordres sanitaires et structurels du bâtiment.

Cette première mission d'évaluation fut suivie, après accord et affinement du projet par Juliette Barbarin, d'une seconde mission plus technique (février 2002) menée par Claire Bergeaud, conservatrice-restauratrice, diplômée du master de conservation préventive et restauratrice conseil du département de Conservation préventive. Elle permit de préciser le contenu du cahier des charges de l'étude prévue : une estimation des volumes des collections afin de définir les surfaces et les équipements de rangement nécessaire, les opérations de traitement à envisager sur les collections (dépoussiérage, désinfestation, mesures conservatoires...), les formations à dispenser au personnel au regard de leur compétence et des besoins du musée et de la collection. Une consultation fut lancée par le musée au printemps et le prestataire choisi en été 2002.

L'ensemble de cette démarche comportait les trois composantes essentielles pour réussir une opération de conservation :

- une volonté et une implication de la responsable avec un objectif simple mais clairement défini : priorité au travail de conservation sur les collections qui allait permettre de sauvegarder les collections et faciliter les activités muséales ;

- une approche méthodique et globale, que Juliette Barbarin a renforcée par des conseils extérieurs ; 
- une approche pragmatique et réaliste : face à un contexte muséal qui n'allait guère évoluer dans la décennie à venir, proposer un plan pluriannuel afin de permettre des réalisations au gré des budgets et du contexte mais dans un cadre de travail bien défini ; dès le début la formation du personnel et son association au chantier avaient été demandées par le musée pour créer une dynamique de projet avec l'équipe, mener des opérations avec ses moyens propres et investir dans une politique de conservation d'avenir.

La définition claire du projet et l'appel à des compétences et à des outils en amont ont été les garanties et le socle de la politique de conservation préventive que le musée Denon a ainsi mise en place.

Roland May

Directeur du centre interdisplinaire de conservation et restauration du Patrimoine (CICRP) de Marseille

Chef du département Conservation préventive au C2RMF de 2000 à 2005

\section{Établissement d'un plan de conservation préventive}

5 Le bâtiment principal du musée $\left(1700 \mathrm{~m}^{2}\right)$ est composé de trois constructions d'époques différentes (1739/

$61830,1866,1907)$, comprenant chacune deux à trois niveaux, et protégées (inscription à l'inventaire supplémentaire en 2001, renforcée en 2004) au titre des Monuments Historiques en fonction de l'intérêt des éléments architecturaux et des aménagements intérieurs subsistants des dépendances d'un ancien couvent et de l'école de dessin. Une salle de Sciences naturelles a été construite dans la cour lors de la dernière extension de 1958. Seule la section Archéologie disposait de réserves extérieures. Les collections de la section Beaux-Arts étaient accumulées tant dans les salles d'exposition que dans deux réserves fermées comprenant également du matériel (emballage, socles, maquettes d'exposition...) ; une troisième ouverte sur un couloir et un escalier menant aux bureaux des archéologues comprenait des grilles à tableaux. La salle de Sciences naturelles était désormais totalement insalubre et la mise hors d'eau ainsi que la mise en conformité électrique de l'ensemble apparaissaient comme le préalable à tout réaménagement. Les collections, pour autant que l'on pouvait alors en juger, ne semblaient pas globalement dans un état catastrophique mais plutôt difficilement accessibles et identifiables. Néanmoins, plusieurs campagnes de désinfestation par anoxie ont permis de juguler la présence d'insectes xylophages dans les châssis, cadres des peintures, le mobilier et les collections ethnographiques. Après les premières formations dispensées à l'ensemble du personnel, d'initiation à l'entretien des locaux, aux principes de manipulation des œuvres, à l'incidence du climat sur l'état des collections, ont été posées les premières bases d'un plan d'urgence.

7 À la suite de deux missions du C2RMF, il est apparu que la mise en œuvre d'un plan de conservation préventive se justifiait : la première étude a porté sur les collections BeauxArts, 10000 œuvres et objets ont ainsi été examinés. 


\section{Étude et plan de conservation préventive des collections de la section Beaux-Arts du musée Denon}

8 Une consultation a été lancée à partir de l'établissement du cahier des charges par le C2RMF. La proposition de Frédérique Vincent a été retenue car elle tenait le mieux compte des objectifs fixés et du budget imparti :

- évaluer l'état de conservation de la collection, afin de servir de base à toute réflexion future ;

- proposer des solutions réalisables avec les ressources humaines et financières existantes afin d'optimiser ou améliorer la situation;

- proposer un plan de conservation préventive.

9 Consultante en conservation préventive et restauratrice d'objets ethnographiques, Frédérique Vincent a organisé une équipe pluridisciplinaire de restaurateurs regroupant les spécialités suivantes: peinture (Françoise Auger-Feige), arts graphiques (Florence Herrenschmidt) et sculptures (Emmanuel Caillé) en raison de la diversité des collections .

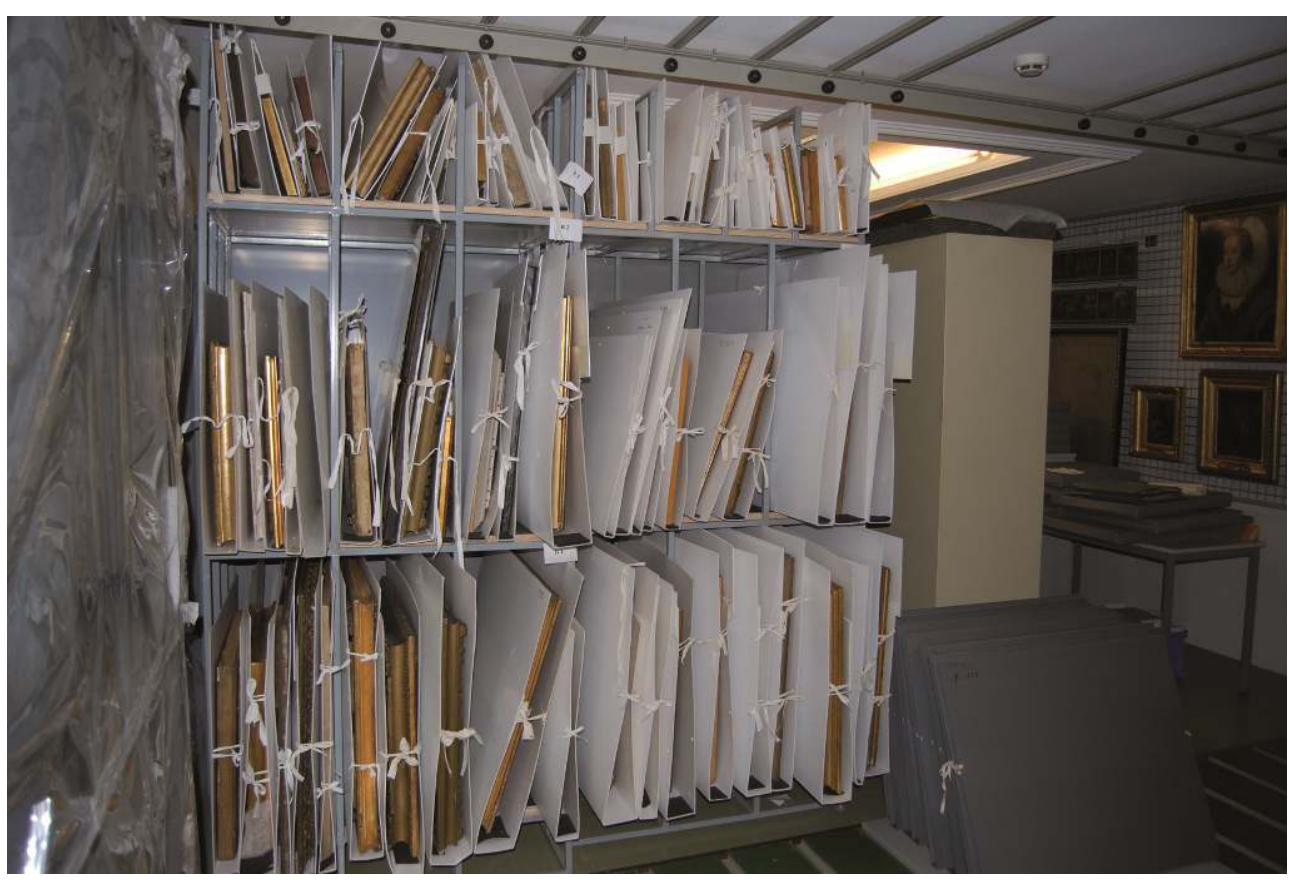

Stockage des peintures dans des porte-folios de polypropylène

(C) MUSÉE DENON/BENOÎT MAISONNEUVE

10 Après l'observation des œuvres et objets dans le musée et l'analyse des données, il est apparu que la collection était globalement en état de conservation stable. Aucune intervention d'urgence n'était nécessaire en dehors d'une intervention de conservation curative (traitement d'un lot de 450 documents graphiques, 8 lots de sculptures et d'une peinture infestés par les micro-organismes) qui devait être menée à court terme. Il est aussi apparu que des restaurations seraient indispensables pour la présentation des œu vres. Par contre, l'étude a montré que d'importantes problématiques de conservation comme l'inaccessibilité et un empoussièrement général - se posaient.

11 Suite à ces conclusions, l'étude a proposé un plan de conservation préventive en trois étapes : 
- à court terme, il semblait qu'un redéploiement et une amélioration du stockage de la collection étaient nécessaires pour la bonne conservation des œuvres, mais aussi pour une utilisation rationnelle et une meilleure connaissance de la collection. Il convenait pour cela de mettre en œuvre un plan de formation du personnel et d'acquérir du matériel de conditionnement et de stockage ;

- la réalisation d'une étude climatique était recommandée en vue de la réorganisation des espaces au sein du musée ;

- pour finir, après l'élaboration du Projet Scientifique et Culturel, un projet architectural à long terme devait être envisagé sur le bâtiment, son contenu et son organisation.

\section{Constitution progressive d'une chaîne opératoire}

12 Au cours d'un premier tri, les collections ont été séparées des autres éléments matériels. L'évacuation d'une réserve à la suite d'un dégât des eaux causé par la réfection d'une toiture a accéléré la mise en place progressive d'une chaine opératoire sur les œuvres. Bien qu'organisées dans le temps par domaines (peintures, documents graphiques, lapidaires, sculptures, objets ethnographiques, mobilier régional), les opérations ont été menées en conservant une vision globale afin de privilégier les mêmes niveaux d'intervention en commençant par les altérations structurelles. Les restaurations à visée esthétique ont fait l'objet d'un plan pluriannuel en fonction des besoins d'exposition.

Les opérations de récolement se sont progressivement ajoutées aux constats d'état détaillés, au dépoussiérage, à l'inventaire, au marquage éventuel, au conditionnement (souvent par étapes en fonction de l'avancement du tri et de l'implantation des structures de stockage). Six réserves ont ainsi été aménagées : dans ces actions encadrées par une équipe de restaurateurs spécialisés il s'est agi de distinguer ce qui relevait de la capacité technique du musée en matière de conservation et des interventions spécifiques des restaurateurs.

14 Les agents d'accueil ont été associés pour la surveillance de l'état des œuvres dans les salles d'exposition, l'entretien du musée et la maintenance de certains équipements (éclairage, humidificateurs...). 


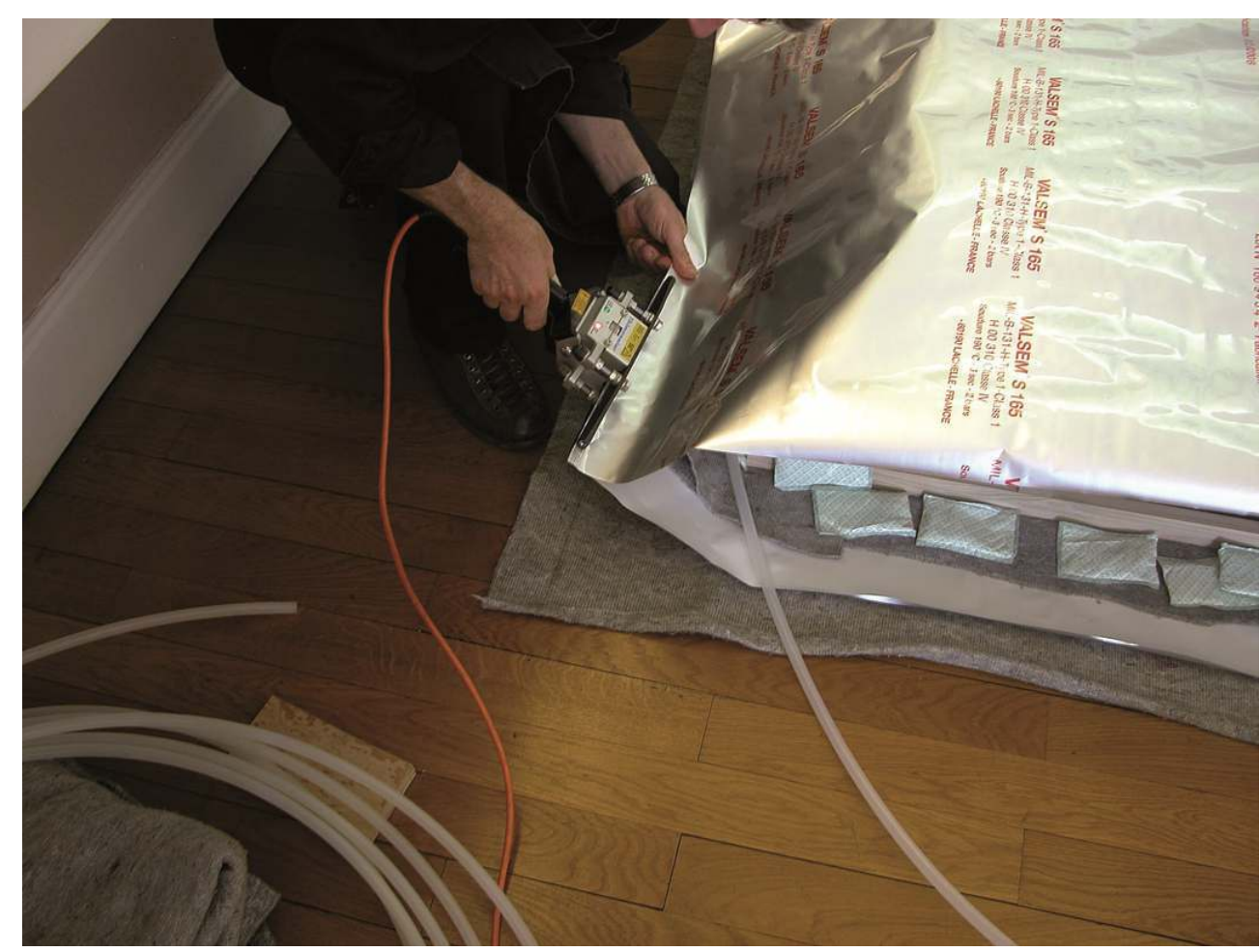

Fabrication d'une poche d'anoxie au musée

(C) MUSÉE DENON/BENOÎT MAISONNEUVE

\section{Formation et encadrement de l'équipe de conservation et des agents techniques}

\section{Peintures}

15 La collection de tableaux comprend une majorité de peintures sur toile, des peintures sur panneaux, quelques peintures sur cuivre, carton, contreplaqué, de tous formats et dont les datations s'échelonnent de la fin $\mathrm{du} \mathrm{XV}^{\mathrm{e}}$ siècle à la fin $\mathrm{du} \mathrm{XX}^{\mathrm{e}}$ siècle. L'évaluation de l'état de conservation des collections du musée Denon, des interventions nécessaires (désinfestation, protections, consolidations, restaurations) et l'observation de leur particulière sensibilité aux variations climatiques pour certaines (peintures sur panneaux flamandes et hollandaises, peintures italiennes rentoilées à la colle de pâte) ont conduit à la construction du processus de formation suivant :

16 - mise en état de conservation avec initiation à l'examen des œuvres (détection des altérations structurelles), aux manipulations, dépoussiérage, emballage ;

17 - protection des feuillures et changement des pattes de maintien des cadres;

18 - aménagement et rangement des réserves en améliorant les grilles, en ajoutant des étagères, des racks, et fabrication de porte-folios pour les œuvres stockées dans ces systèmes. 


\section{Arts graphiques}

19 Les 3000 documents graphiques (dessins anciens XVII ${ }^{e}$-XIX ${ }^{e}$ siècles, fonds Denon, estampes essentiellement XIX ${ }^{\mathrm{e}}$ siècle, miniatures sur ivoire) ont fait l'objet d'un premier tri dessins/estampes/miniatures/matrices d'impression/imprimés/autographes, puis par techniques de dessin (sèches, humides) afin de définir le conditionnement (passe-partout ou pochettes) dans quatre formats (trois formats de boites de conservation, un grand format dans des tiroirs de meubles à plan).

Les protocoles pour le conditionnement et l'exposition des œuvres ont été exécutés en interne par l'équipe sous la responsabilité du restaurateur qui a introduit la méthode suivante :

- principes généraux sur le papier : qualités ; manipuler, décadrer, réaliser les constats d'état des documents graphiques;

- conditionnement: mise sous pochettes après dépoussiérage, préparation de lots à traiter entre deux formations ;

- achat de petits matériels, de conditionnements classiques (boîtes, cartons conservation et papier pour pochettes) ou spécifiques (classeurs à pochettes polyester pour assignats et petits documents imprimés...).

Une centaine de miniatures sur ivoire a fait l'objet de deux campagnes spécifiques par un restaurateur spécialisé qui a su sensibiliser et associer les agents du musée au conditionnement de ces collections, préalablement et postérieurement à son intervention.

\section{Objets}

21 Une formation initiale des agents du musée avec des personnels du musée Niépce et des musées de Bourgogne dans le cadre d'une formation du Centre national de la Fonction publique territoriale, organisée au musée, a été l'occasion de présenter les principes gé néraux du conditionnement des collections muséographiques, les matériaux de conservation (avantages/inconvénients) et les techniques de mises en œuvre. Les formations se sont ensuite poursuivies au rythme de plusieurs sessions de 2 à 3 jours par an.

De natures très diverses (objets archéologiques de collection, objets d'arts décoratifs, objets ethnographiques nationaux et extra-européens, textiles, matériels techniques et scientifiques...) ces collections ont été conditionnées dans des supports (mousses, boîtes, armatures...) fabriqués à l'occasion, adaptés à la dimension des objets et aux matériels de stockage (armoires, rayonnages, structures à bacs plastiques...). 


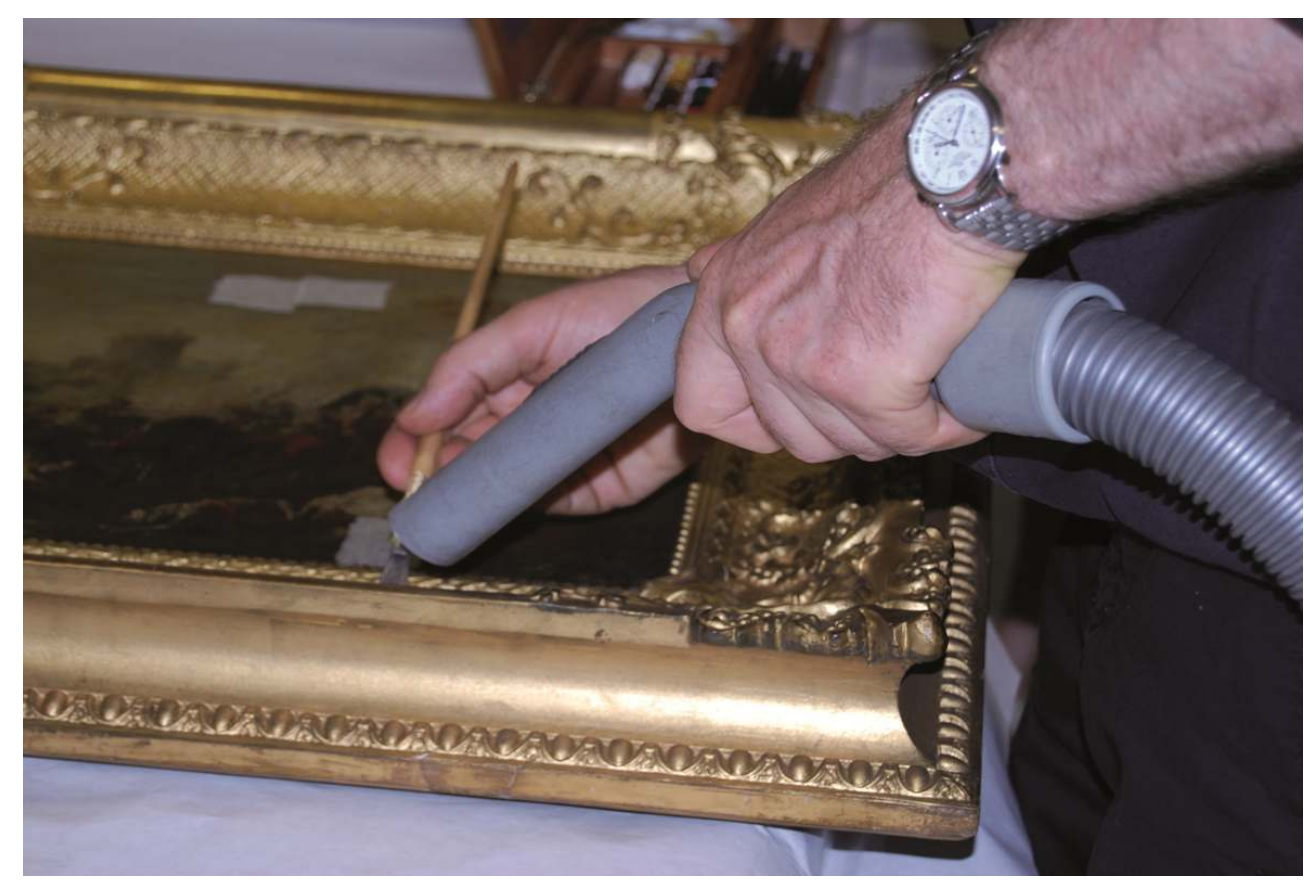

Formation au dépoussiérage des cadres

(c) CRCOA/AUBERT GÉRARD

\section{Soclage}

La réalisation de supports en raison de la nature et/ou l'état de certains objets s'avère particulièrement efficace pour les objets archéologiques. Afin de pouvoir les réaliser selon les directives du restaurateur, les agents du musée ont intégré :

- le rôle et la nécessité du soclage pour le maintien de l'objet sans contrainte ;

- l'apport du socle pour la compréhension de l'objet dans un contexte de présentation.

La connaissance de la nature des matériaux constitutifs des supports (métaux, verre, verres synthétiques, bois), de leurs qualités mécaniques et des éventuelles contraintes physico-chimiques a précédé la mise en place d'un atelier de fabrication (laiton formé et soudé). Les différentes techniques de réalisation, les finitions utiles à la protection des points de contacts support/objet ainsi que l'évaluation des risques et des conditions d'installation en vitrine ont été abordés.

Cette approche des objets archéologiques se poursuit sur les objets en trois dimensions et sur les sculptures afin de les exposer d'une manière sécurisée et esthétique.

\section{Restauration}

Le chantier des collections a été organisé afin de réaliser les traitements de mise en état de conservation :

- pose de protections de surface sur les zones de soulèvements, stabilisation des déchirures, amélioration des maintiens sur les châssis pour les peintures ;

- démontage des documents graphiques: assainissement et élimination des matériaux polluants ; 
- dessalement de bas-reliefs, remplacement de structures métalliques internes de sculptures, traitements biocides.

Enfin des interventions plus importantes de conservation ou de restauration ont été exécutées sur les œuvres destinées à l'exposition.

\section{Étude de climat}

27 Si un constat global avait été préalablement dressé afin d'engager les travaux les plus urgents concernant la mise hors d'eau de certaines zones du musée, les désordres constatés sur les œuvres notamment au cours de l'étude en conservation préventive signalaient des anomalies qu'il convenait de rechercher au niveau du bâtiment. Le climat du musée Denon de Chalon-sur-Saône a donc été étudié en 2004 à partir d'enregistrements réalisés en 2003 et 2004.

Trois capteurs-enregistreurs de température et d'humidité relative ont circulé dans le musée de salle en salle durant dix-huit mois, sur des cycles de deux mois environ, un capteur étant placé à l'extérieur. L'interprétation des données brutes recueillies reflétait le climat intérieur du musée. Il s'agissait de mettre en évidence les particularités du climat intérieur, de les cor-

réler avec les données architecturales du bâtiment et l'état de conservation des collections. Le musée Denon étant un musée municipal à budget réduit, il fallait écarter d'emblée certaines solutions onéreuses (climatisation du bâtiment, aménagements conséquents de l'architecture...). L'étude de climat devait donc établir des préconisations de solutions gérables en interne.

Les propositions qui ont découlé des conclusions de cette étude sont de trois types :

- une meilleure gestion des espaces en fonction de la sensibilité des collections aux variations de la température et de l'humidité relative ;

- des mesures peu coûteuses, réalisables en interne ou par les services techniques de la Ville, pour des améliorations ponctuelles du climat de certains espaces du bâtiment ;

- des travaux plus importants tels que l'amélioration du système de chauffage, la mise en place d'un sas d'entrée... visant à remédier à des désordres climatiques communs à un groupe d'espaces ou au bâtiment tout entier. 

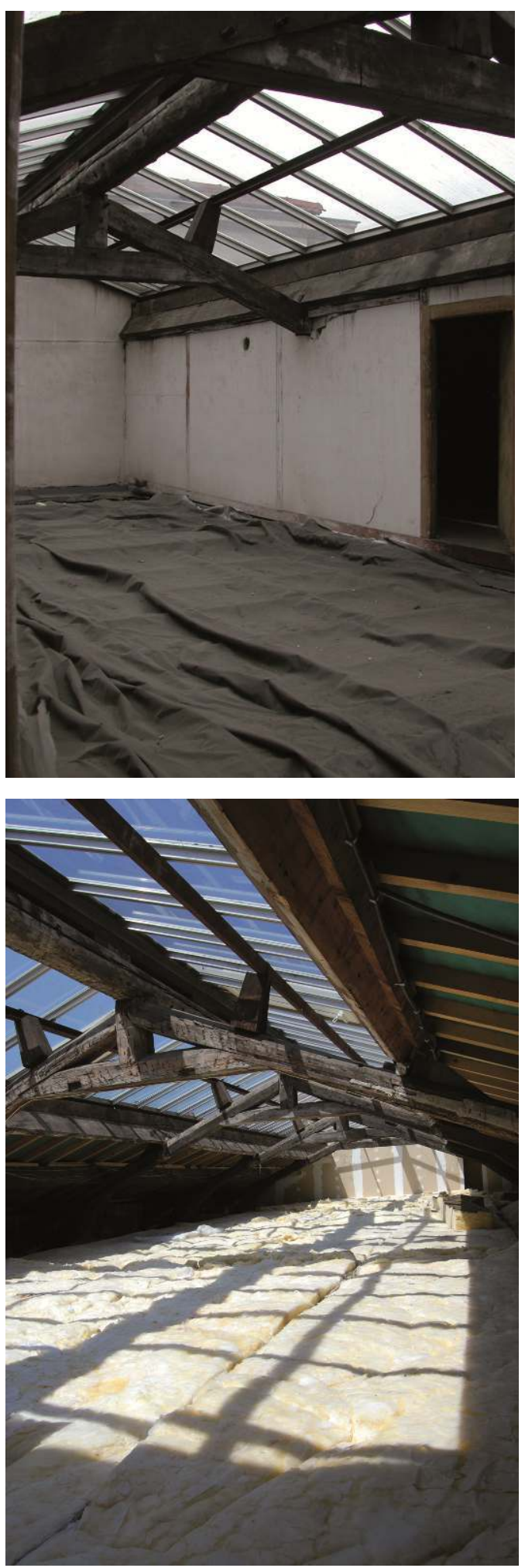

Verrière au-dessus de la salle Denon avant travaux en 2002 et une fois isolée après la réfection du toit. (c) Frédérique Vincent

(c) Musée Denon/Mathilde Ujeda

31 À l'occasion de la rédaction de cet article un bilan a été dressé avec les restaurateurs chargés de l'étude: des travaux de réfection des toitures qui s'inscrivaient par chance dans un programme global d'entretien des bâtiments municipaux, ont assuré une 
meilleure isolation. Des mesures ponctuelles dans les salles et les réserves ont été prises ainsi que la constitution d'un parc d'humidificateurs. Le nombre de capteurs a été augmenté mais pour assurer le suivi du climat, il est difficile de multiplier les tâches au sein d'une équipe qui assume à la fois des missions de fonds (chantier des collections), de maintenance, de préparation et de réalisation des événements (expositions, manifestations...). Un renfort pendant les périodes d'événementiel pourrait maintenir la vigilance requise dans un environnement où les actions correctives sont à estimer et à effectuer au cas par cas.

L'autre difficulté rencontrée est la quasi impossibilité de réaliser des travaux d'ensemble notamment sur l'installation de chauffage. Ces derniers sont considérés comme des travaux d'entretien et non comme des mesures indispensables pour la conservation du bâtiment et des collections. La limitation des investissements à des mesures ponctuelles ne permet pas d'appréhender l'importance décisive de certaines améliorations comme c'est le cas lors d'une planification pluriannuelle d'un projet de rénovation.

Mais l'étude climatique a permis d'identifier précisément les facteurs d'altération, de projeter des travaux et des études complémentaires ainsi que des interventions de restauration ou au contraire de différer des restaurations (panneaux peints) et la présentation de certaines collections (miniatures sur ivoire). Il a aussi été possible d'affecter certains espaces à certaines collections (par exemple le mobilier régional dans une salle non chauffée l'hiver). Globalement on constate une réduction de l'amplitude des variations et un certain déphasage de celles-ci. Mais il n'a pas été remédié aux inconvénients du système de chauffage/ventilation et les périodes de plein été et de plein hiver restent critiques.

Enfin, l'évolution récente de l'équipe technique permet d'envisager le maintien d'un calendrier de relève des capteurs et d'analyses des données ainsi que la modernisation du système de relève des données.

\section{La gestion des espaces : aménagement de réserves et redéploiement des collections}

\section{Sculptures et éléments lapidaires}

Les 300 sculptures et éléments lapidaires ont été répartis dans trois réserves dont une en extérieur. La première opération a consisté à déposer et déménager dans la cour du musée sous un abri aménagé une vingtaine d'éléments lapidaires. Le musée ne disposant d'aucun appareil de levage ni d'aucune expérience dans la manutention d'éléments lourds, il a été fait appel à un transporteur ainsi qu'à un restaurateur pressentis séparément pour une réalisation conjointe de l'opération. Ce dernier a fourni les informations pour la préparation des locaux, pour le transfert des éléments et pour la structure de réception des éléments. D’abord déconcerté par cette collaboration imposée, le restaurateur en a tiré le meilleur parti, du point de vue de la conservation des œuvres comme des responsabilités respectives. L'équipe technique du musée, en les assistants, a intégré les connaissances essentielles dans la manutention des objets lourds. 


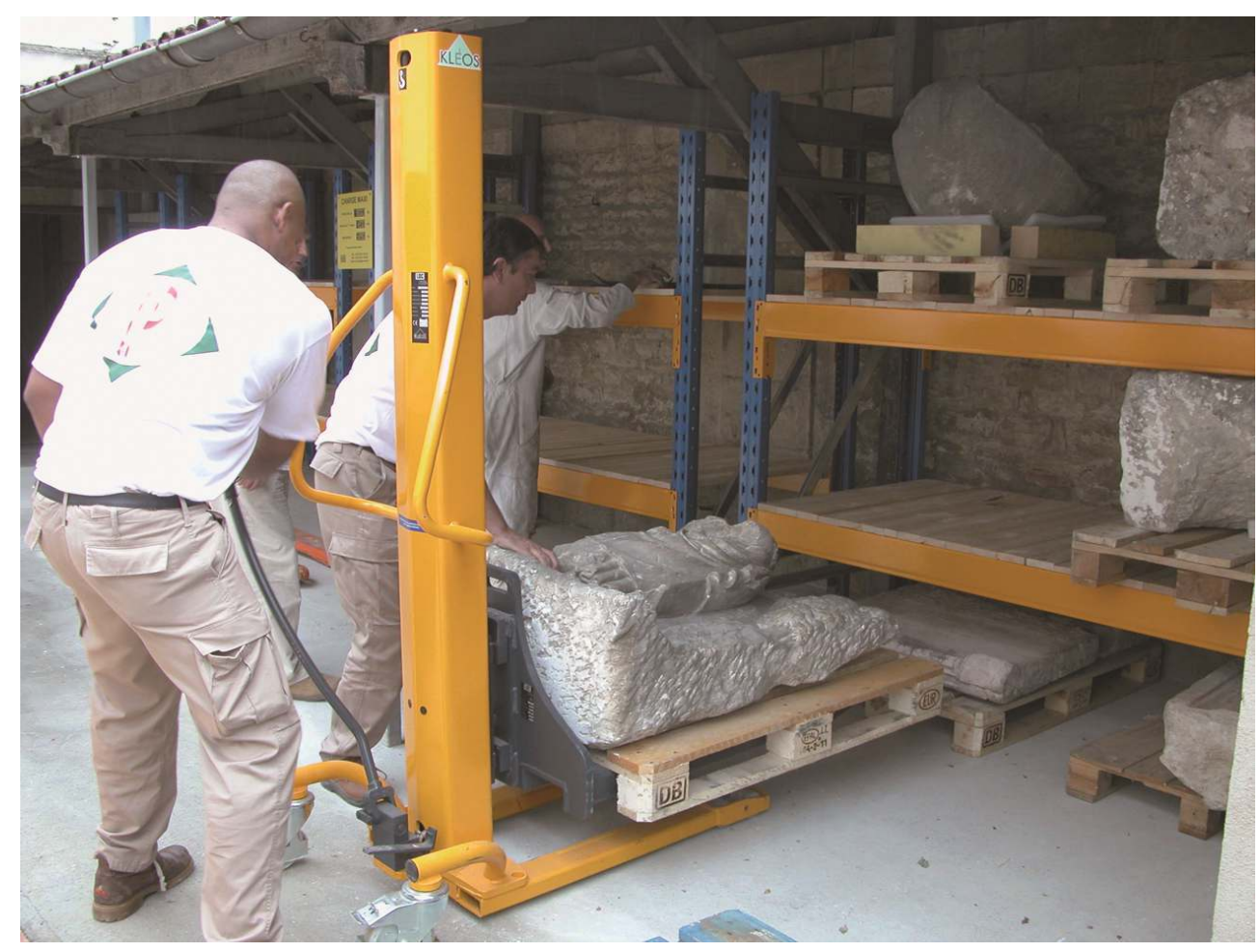

Déménagement des collections de lapidaires

(C) MUSÉE DENON/MATHILDE UJEDA

36 À la suite de cette expérience, d'autres éléments lapidaires ont pu rejoindre les premiers et deux réserves de sculptures ont été aménagées regroupant les bois et les terres cuites et les métaux pour l'une, les pierres pour la seconde.

\section{Mobilier régional}

Cinq armoires et cinq buffets à deux corps étaient présentés dans une salle du premier étage du musée. Une verrière, présente sur une grande surface du plafond, générait des variations importantes du climat avec des chutes importantes du taux d'hygrométrie.

La présentation de ces meubles dans une salle au rez-de-chaussée a donc été envisagée. Après mesure et enregistrement des données climatiques de chaque salle, il a été décidé de procéder au déménagement, sans démontage complet, des meubles préalablement désinsectisés, à une période où les climats étaient très proches dans les deux salles d'exposition.

\section{De la réserve à l'exposition}

39 Après le transfert du mobilier régional et l'isolation du toit et de la verrière, la salle d'exposition permanente a été destinée à la présentation d'un cabinet d'arts graphiques consacré à l'œuvre de Vivant Denon.

40 À l'origine constituée d'une centaine de dessins et estampes, la collection Denon comprend aujourd'hui plus de 600 numéros grâce aux acquisitions et aux dons effectués depuis une dizaine d'années avec l'aide d'un comité scientifique présidé par Pierre Rosenberg, de l'Académie française, président-directeur honoraire du musée du Louvre. 
La fragilité des collections graphiques et des livres anciens nécessitait une réflexion particulière, conduite avec une restauratrice spécialisée, pour la conception d'une salle d'exposition permanente. Un système d'éclairage filtré et temporisé a permis d'allonger la durée d'exposition des documents dans le cadre d'une rotation régulière et de sélectionner des œuvres de référence exposées plus durablement dans des meubles à tiroirs.

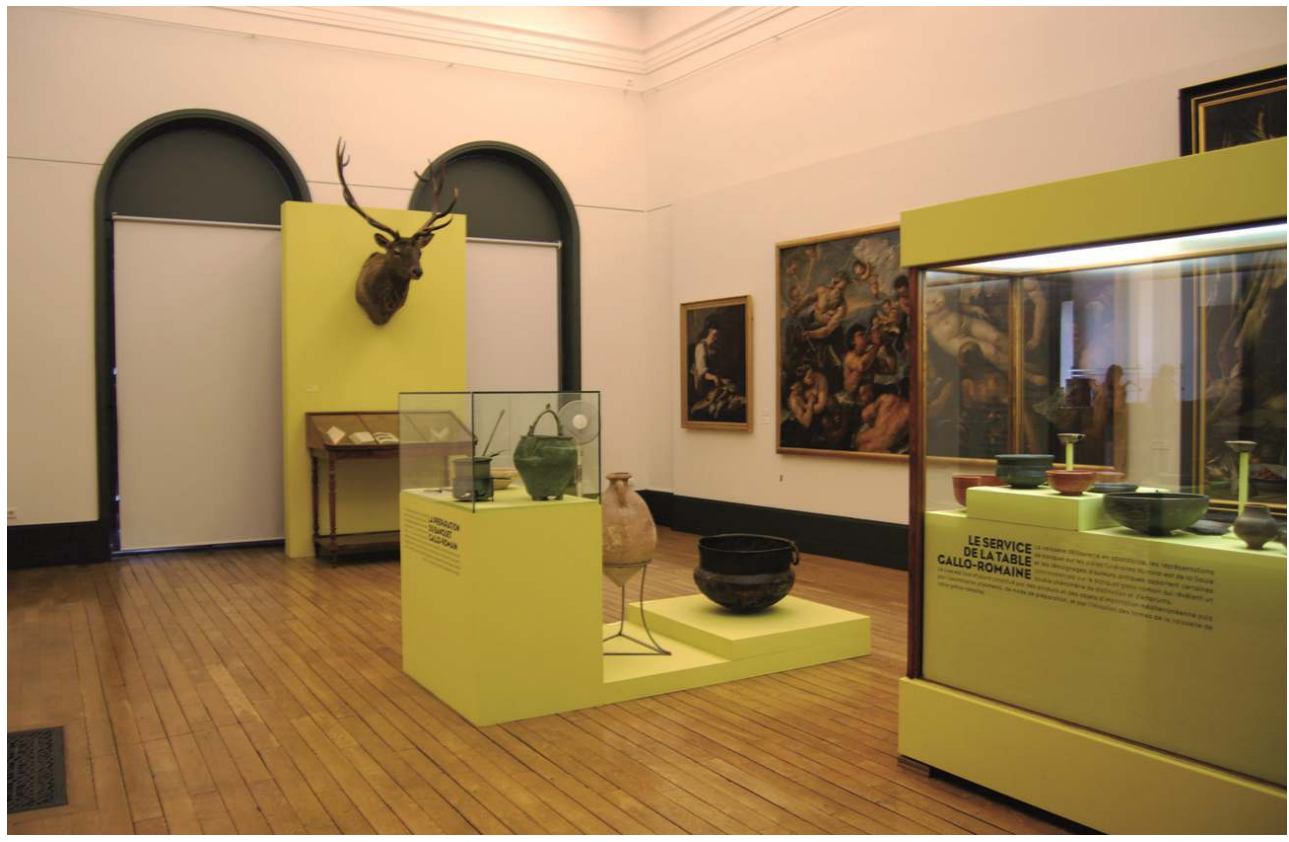

Exposition Banquets, scènes de genre, vie domestique, 2013

(C) MUSÉE DENON/MATHILDE UJEDA

\section{Perspectives}

Grâce à une dizaine de jours de formation par an, l'aménagement des réserves et le conditionnement des collections se sont déroulés sur un rythme régulier malgré l'évolution de la composition des équipes. Les compétences acquises au cours de ces opérations ont également permis la production d'expositions temporaires et la réfection de salles de présentation permanentes (salle gallo-romaine, salle Denon).

La principale difficulté - ne pas occasionner d'emprise spatiale supérieure à l'existant - a été résolue par la concertation entre les équipes internes et externes notamment en faisant évoluer les principes de stockage du conditionnement individuel au stockage en série, ou en opérant des regroupements dans des espaces communs en fonction de l'encombrement des collections : objets conditionnés par étagères dans des armoires thé matiques, définition d'une grande réserve peintures/objets, aménagement d'une petite réserve de sculptures (bois, plâtres, terres cuites), textiles et matériel scientifique dans des structures indépendantes mais concentrées.

La communication au public de ces actions est intervenue par voie de presse locale, de présentations pendant les Journées du Patrimoine ou de conférences, de visites des réserves.

Si l'accessibilité des collections beaux-arts en réserve est effective pour l'équipe du musée, un travail conséquent sur les collections archéologiques et le bâtiment reste à 
mener. Une étude sur les collections archéologiques vient d'être réalisée afin de poursuivre la mise en œuvre du plan de conservation préventive.

La nouvelle écriture du Projet Scientifique et Culturel du musée ne peut faire l'économie de la conservation préventive comme méthode de gestion et d'utilisation rationnelle des collections et de ce qui n'est pas encore traité : la reconnaissance du bâtiment dans son ensemble comme lieu d'exposition et de conservation, la nécessité de réserves extérieures efficientes en termes de conservation et d'usage des collections. Loin de constituer des dépenses superfétatoires et des interventions définitives, la conservation préventive constitue une dynamique de gestion et de diffusion des collections, s'inscrivant ainsi légitimement dans le volet culturel des projets de développement durable 3 .

L'auteur tient à remercier la ville de Chalon-sur-Saône, la DRAC de Bourgogne, la société des Amis du musée Denon, le comité scientifique du musée Denon et son président, Pierre Rosenberg, le CNFPT Bourgogne et Florence Bertin, Jean-Louis Biard, Gérard Blanc, François Cheval, Aubert Gérard, Florence Herrenschmidt, Frédéric Ladonne, Véronique Langlet-Marzloff, Lionel Lefèvre, Gilles Mantoux, Isabelle Maquaire, Roland May, Berndt Pappe, Estelle Rebourg, Alain Renard, Juliette Rollier, Annabelle Simon, Colette VicatBlanc.

\section{NOTES}

2. May, R. Regards sur la conservation préventive dans les musées de France, La Lettre de L'OCIM, n 138, novembre-décembre 2011, p. 6.

3. Guillemard, D. D'une simple attitude à une discipline, l'évolution de la notion de prévention, Techné, n 34, 2001, p. 10.

1. Ont collaboré à l'écriture de cet article : Françoise Auger-Feige, restauratrice de peintures ; Isabelle Boiché, consultante en conservation préventive; Bérengère Chaix, restauratrice de documents graphiques; Emmanuel Desroches, restaurateur de sculptures; Catherine Lebret, restauratrice de peintures, consultante en conservation préventive; Christian et Françoise Morin, restaurateurs de peintures; Thierry Palanque, restaurateur de supports bois, mobilier, socleur; Frédérique Vincent, restauratrice d'objets ethnographiques, consultante en conservation préventive.

\section{RÉSUMÉS}

Dans le cadre de la réorganisation du musée Denon de Chalon-sur-Saône engagée depuis 2000, un diagnostic établi par le Centre de Recherche et de Restauration des Musées de France a montré qu'il était indispensable de concevoir un plan de conservation préventive: l'auteur et les différents intervenants extérieurs décrivent les étapes de cette opération depuis l'aménagement 
des réserves jusqu'au redéploiement des collections en passant par les interventions en conservation-restauration du chantier des collections ${ }^{1}$.

\section{AUTEUR}

\section{JULIETTE BARBARIN}

Juliette Barbarin qui a coordonné cet article est attachée de conservation du patrimoine au musée Denon à Chalon-sur-Saône. juliette.barbarin@chalonsursaone.fr 